\title{
Cost of Oncology Drugs in the Middle- Eastern Country of Lebanon: An Update (2014-2016)
}

Fadia Elias

Ibrahim R. Bou-Orm

Salim M. Adib

Selim Gebran

Anthony Gebran

Walid Ammar

Author affiliations and support information (if applicable) appear at the end of this article. Corresponding author: Ibrahim R. Bou-Orm, MD, MPH, Ministry of Public Health, Beirut, Lebanon; e-mail: ibrahim. bouorm@gmail.com.

Purpose This study aims to evaluate trends in the increasing costs of oncology drugs procured by the Lebanese Ministry of Public Health (MOPH) between 2014 and 2016 and to assess the impact of the introduction in mid-2015 of new immunotherapy drugs for the treatment of lung cancer on the overall and specific costs of that treatment.

Methods A secondary analysis of data from the MOPH Cancer Drug Scientific Committee data base was conducted using a total of 18,133 cancer files between 2014 and 2016.

Results Over the 3-year period, about \$140 million (USD) was spent on cancer drugs by the MOPH free cancer drug dispensing program. The expenditures increased by $27 \%$ after immunotherapy was phased in. The average cost of drugs per patient per year measured across all cancer types increased from $\$ 7,000$ in 2014 to $\$ 8,400$ in 2016 . Trastuzumab, approved for treating human epidermal growth factor receptor 2-positive breast cancer ranked first in total expenditures for 2014-2015. By 2016, two new immunotherapy drugs had topped the list: pembrolizumab ranked first and nivolumab ranked third, representing $64 \%$ of the total cost of lung cancer treatment and approximately $19 \%$ of the total yearly budget; beneficiaries represented only $3 \%$ of all patients.

Conclusion This update documents the increasing financial impact of newer cancer drugs on the procurement process in the middle-income country of Lebanon. The trend is aligned with the financial burden of cancer drugs worldwide, which calls for a collaborative global response to this crisis.

@ 2018 by American Society of Clinical Oncology Licensed under the Creative Commons Attribution 4.0 License

\section{INTRODUCTION}

Lebanon is facing a mounting financial and health care burden from cancer, which doubled in incidence in the last 50 years and is associated primarily with the steady aging of the population. ${ }^{1,2}$ To avoid catastrophic financial consequences for households as a result of out-of-pocket expenditures for cancer treatment, the Ministry of Public Health (MOPH) has been providing free oncology drugs since 1999 to all Lebanese patients who have no formal health coverage; almost half the Lebanese population of about 4.3 million are in this category. ${ }^{3} \mathrm{~A}$ previous assessment of $\mathrm{MOPH}$ data between 2008 and 2013 showed a steady increase in the average costs of cancer drugs per patient from $\$ 4,863$ to $\$ 7,803$ (note that US dollars are used throughout). Expensive targeted therapy has been approved by the MOPH for treating the majority of cancer types for several years. ${ }^{4}$ More recently, in 2015, immunotherapy was also approved for treating some types of cancer. Although this advance has a major positive impact on patients' survival, it brings an additional devastating economic burden to the population and the health care system. ${ }^{5}$

This article updates the previously published figures on the costs of cancer drugs incurred by the Lebanese MOPH from 2014 to 2016. It specifically assesses the impact of the introduction in mid-2015 of two new immunotherapy drugs for treating lung cancer, pembrolizumab (Keytruda) and nivolumab (Opdivo), on the overall cost of cancer drugs and the specific cost of drugs for lung cancer.

\section{METHODS}

This is a secondary analysis of data from the MOPH Cancer Drug Scientific Committee data base. A total of 18,133 cancer files on patients who had received approval for drug treatment coverage for years 2014 to 2016 were included in the analysis. The researchers analyzed 


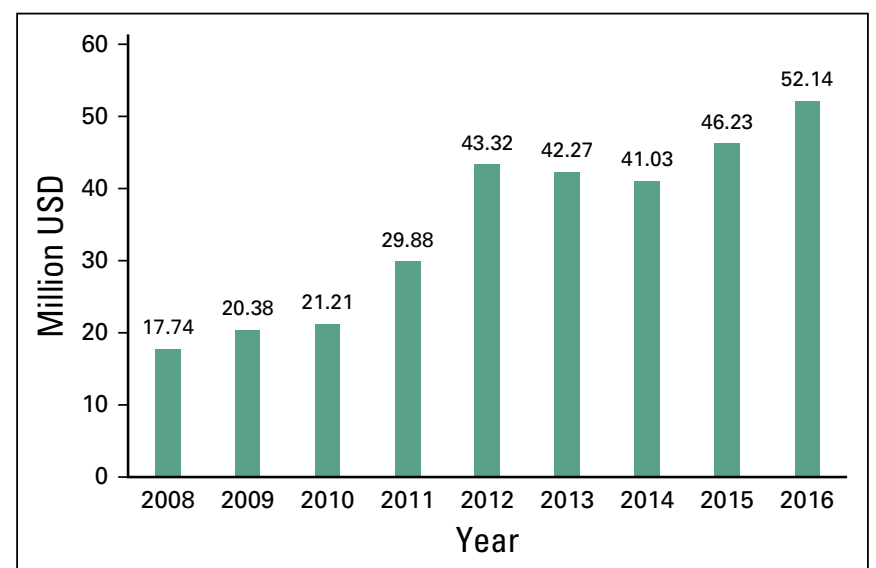

Fig 1. Cost of cancer drugs procured by the Lebanese Ministry of Public Health from 2008 to 2016.

Fig 2. Number of patients most expensive cancer drug (trastuzumab, and yearly average drugs cost from 2008 to 2016. the total number of patients with cancer) were computed for each year. Expenditures by drug type were tabulated for the most expensive drugs. The changes in prices for selected cancer drugs for two time periods (2011 to 2013 and 2013 to 2015) were graphed to assess the impact of MOPH policies on the pricing of these drugs.

\section{RESULTS}

\section{Total Financial Burden of Cancer Drugs Incurred by MOPH}

Over the 3-year period, a total of approximately $\$ 140$ million was spent by the MOPH free cancer drug dispensing program. Even though the bevacizumab, imatinib, and rituximab) prices were decreased by an average of 35\% in 2016 through tough procurement negotiations, the amount spent was slightly over $\$ 52$ million in 2016 compared with $\$ 41$ million in 2014 . This increase of almost $27 \%$ followed the phasing in of immunotherapy drugs. Figure 1 shows trends in drug expenditures using data available for analysis since 2008, with increases linked to the introduction of the newer, more expensive drugs. The number of patients covered by the free cancer drug dispensing program was relatively stable at approximately 6,000 each year, increasing from 5,857 patients in 2014 to 6,207 patients in 2016. Consequently, the average drug cost per patient, measured across all cancer types, increased from $\$ 7,000$ in 2014 to $\$ 8,400$ in 2016 (Fig 2).

\section{Costs by Type of Drug}

As in previous years, trastuzumab (Herceptin), which is approved by MOPH for treating human epidermal growth factor receptor 2 (HER2)positive breast cancer, ranked first in total expenditures for 2014 and 2015. Imatinib (Glivec), which is used for treating chronic myeloid leukemia, was in second place in both 2014 and 2015. New immunotherapy drugs for lung cancer approved in mid-2015 topped the list in 2016: pembrolizumab ranked first and nivolumab ranked third, and $\$ 6.5$ million was spent on their procurement, which represents almost $64 \%$ of the total cost of lung cancer treatment. Table 1

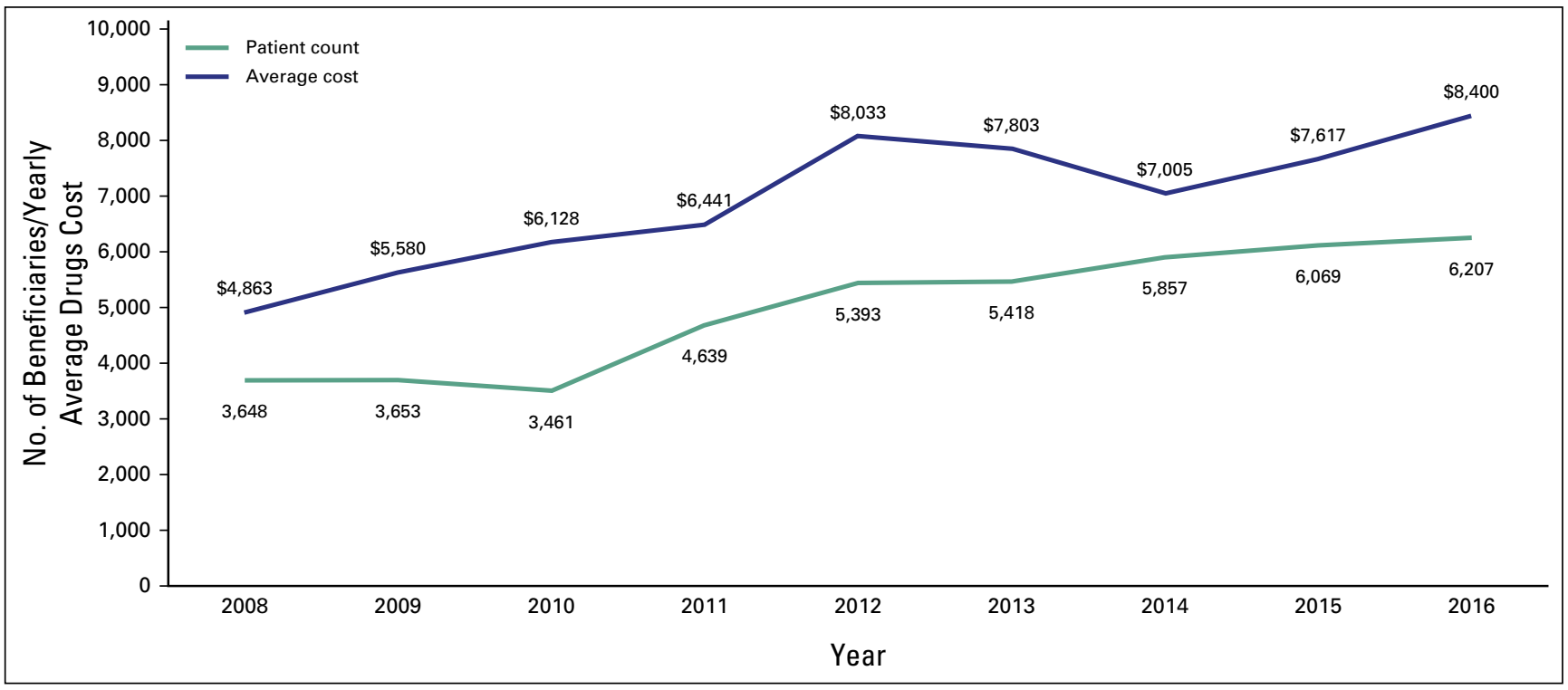


ranks the top 5 most expensive drugs in terms of total yearly spending.

\section{Changes in the Cost of Lung Cancer Treatment}

According to the latest data from the National Cancer Registry (2015), lung cancer is the second most common cancer type among males (13.1\%) and third most common cancer type among females (5.9\%). ${ }^{2}$ In 2016, the two immunotherapy drugs (pembrolizumab and nivolumab) accounted for approximately $19 \%$ of the total yearly expenditures on cancer drugs. The beneficiaries were 192 patients (24\%) of a total of 797 patients with lung cancer. This group of patients represents only $3 \%$ of all cancer beneficiaries. The total cost of drugs for treating lung cancer after the introduction of immunotherapy changed, as expected. ${ }^{6}$ It increased from approximately $\$ 3$ million in 2012 to approximately $\$ 10$ million in 2016. The average annual cost of drugs per patient with lung cancer increased by $160 \%$ between $2012(\$ 5,000)$ and $2016(\$ 13,000)$.

\section{DISCUSSION}

These new figures document the continuously increasing financial impact of newer cancer drugs on the procurement process in the middleincome country of Lebanon. The increase signals a major financial problem in several developing and developed nations. In the United States, Medicare (the national health insurance program for older adults) spending on Part B drugs (the category dominated by cancer treatment drugs) increased from \$3 billion in 1997 to $\$ 25.7$ billion in 2015 (8.6 times), whereas overall Medicare spending increased from $\$ 210$ billion to $\$ 638$ billion (3 times). 7,8

In Lebanon in particular, the doubling of total costs of oncology drugs over a short 5-year span could not have come at a more difficult time. Since 2011, Lebanon has been facing an influx of refugees from civil wars in Syria and Iraq, which inflated its population by at least 30\% for an almost stable population of Lebanese nationals of approximately 4.3 million people. ${ }^{9}$ The tremendous costs of treatment have meant that those refugees previously diagnosed with cancer or diagnosed while in Lebanon could not be easily integrated into the free cancer drug dispensing circuits currently serving only Lebanese patients. The resulting human suffering is putting serious strains on practitioners who find themselves unable to treat those patients adequately.

Although many new cancer therapies have changed the course of the disease, Mailankody and Prasad ${ }^{10}$ reported in a research letter, based on data from the United States, that an independent relationship exists between the price of cancer drugs and their impact on patients' health; they concluded that the irrational pricing mechanism is driven mainly by market dynamics. Moreover, a recent publication by Kumar et al ${ }^{11}$ showed that only $19 \%$ of cancer drugs approved by the US Food and Drug Administration met the ASCO goal of achieving significant clinical outcomes in terms of overall survival, even though the prices are extremely high in many cases. There is an urgent global need for pharmaceutical companies to be held accountable for their pricing practices and to acknowledge their responsibility for human rights. ${ }^{12}$ Disclosure of all costs related to drug discovery, research and development, and marketing would allow transparency and accurate evaluation of the price of a drug in the market compared with the costs needed for its existence. Moreover, international scientific societies are invited to assess the end points of cancer clinical trials to move toward value-based cancer care. ${ }^{13}$

This article is yet another plea for an international approach to limiting the relentlessly increasing costs of cancer drugs that are generating inequity especially in low-income nations where the affordability of both brand-name and generic drugs is lower. ${ }^{14,15}$ The experience in Lebanon shows that measures by one country to decrease the prices of cancer drugs usually have insignificant effects unless they came from the experiences of other countries. Lebanon, a country with a small market, has been able to successfully reduce the prices of several oncology drugs by comparing local prices with those in other countries to generate evidence for negotiating possible discounts. The MOPH established regulations in 2015 requiring that pharmaceutical companies disclose the reduction of any export price in comparable countries within a 3-month interval. The effects of these policies on the prices of selected cancer drugs are shown in Figure 3. The MOPH has recently started to negotiate the price of oncology drugs with drugs companies even before deciding to 


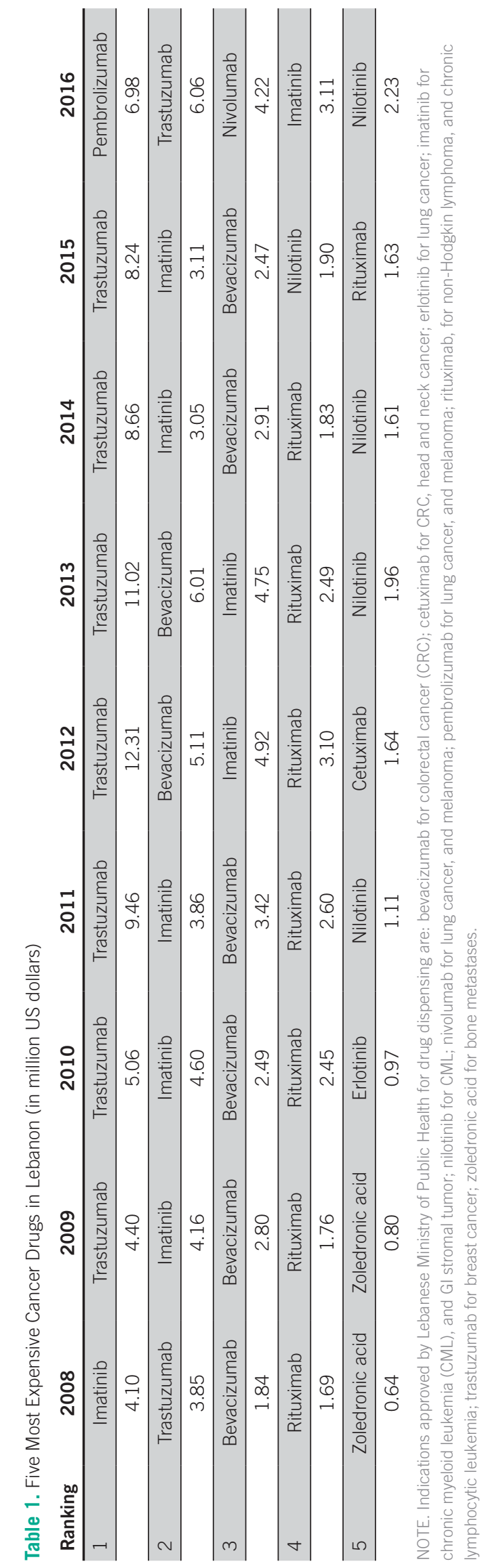




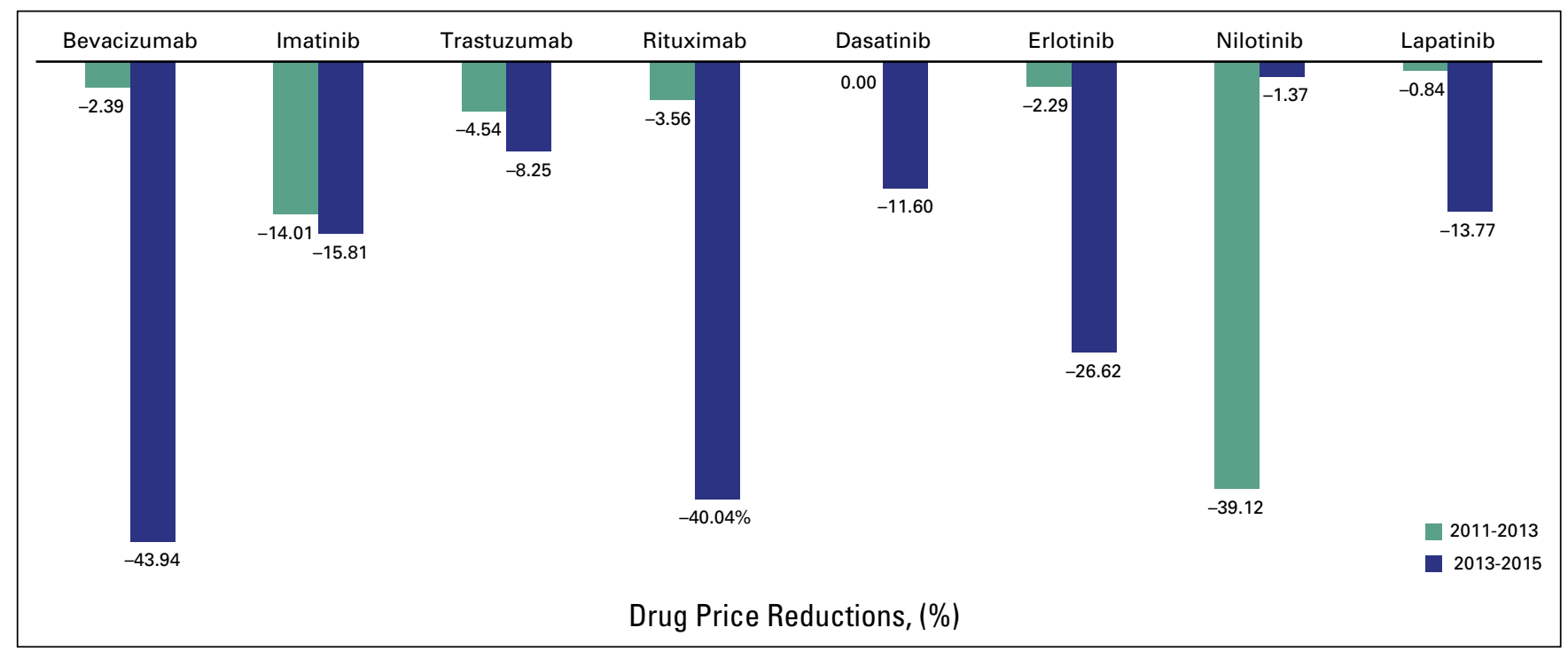

Fig 3. Drug price reductions for selected cancer drugs in Lebanon from 2011 to 2015. provide them free of charge to eligible patients. Inspired by the United Kingdom's National Institute for Health and Care Excellence conducting a cost-effectiveness analysis to include a new drug in its guidelines, and the recent United Kingdom's National Health Services policy of renegotiating prices to make them more affordable, ${ }^{16}$ the $\mathrm{MOPH}$ is reconsidering its policy to ensure the financial sustainability of its free cancer drug dispensing program.

Despite all that, the MOPH is still struggling with high demand for brand-name drugs, even though there are generic competitors or biosimilars. Although these alternatives would improve access to cancer treatment, ${ }^{17}$ decision makers need to understand their importance and not be lenient toward the preferences of providers and manufacturers of original drugs, regardless of their prices. Affordability of cancer treatment should be at the center of decision making for oncology professionals as well. The ASCO position statement on this matter clearly includes drug prices in the core of value-based cancer care and calls upon physicians for appropriate use of oncology drugs, taking into consideration both clinical and financial perspectives..$^{13}$ The experience from Lebanon endorses this suggestion because the common practice in

\section{AUTHOR CONTRIBUTIONS}

Conception and design: Fadia Elias, Ibrahim R. Bou-Orm, Salim M. Adib, Walid Ammar

Administrative support: Salim M. Adib, Walid Ammar

Collection and assembly of data: Fadia Elias, Selim Gebran, Anthony Gebran, prescribing cancer drugs is somehow driven by financial incentives for health care providers, not only by clinically meaningful outcomes, according to regularly updated national guidelines. ${ }^{18}$

In conclusion, tackling the financial burden of cancer in Lebanon cannot be only based on the cost containment of the procurement of drugs and the subsidization of health services. Initiatives toward primary prevention such as tobacco control, secondary prevention such as screening and early detection, and tertiary prevention by improving the quality of palliative care should be continuously supported. The national tobacco control law, created in 2011, urgently needs to be re-enforced, especially by implementing the portion of that law that bans smoking in enclosed public places. Screening for breast cancer (initiated in 2002) and screening for colorectal cancer (expected in 2018) must be continuously supported by policymakers as being cost-effective. Finally, escalating palliative care initiatives in health facilities in Lebanon would help reduce the use of treatment regimens at the end of life and result in better quality of life for patients with cancer.

DOI: https://doi.org/10.1200/JG0.17.00179

Published online on jgo.org on April 2, 2018.

Data analysis and interpretation: Fadia Elias, Ibrahim R. Bou-Orm, Salim M. Adib, Anthony Gebran, Walid Ammar

Manuscript writing: All authors

Final approval of manuscript: All authors

Accountable for all aspects of the work: All authors 
AUTHORS' DISCLOSURES OF

POTENTIAL CONFLICTS OF INTEREST

The following represents disclosure information provided by authors of this manuscript. All relationships are considered compensated. Relationships are self-held unless noted. I = Immediate Family Member, Inst = My Institution. Relationships may not relate to the subject matter of this manuscript. For more information about ASCO's conflict of interest policy, please refer to www. asco.org/rwc or ascopubs.org/jco/site/ifc.

Fadia Elias

No relationship to disclose
Ibrahim Bou-Orm

No relationship to disclose

Salim Adib

Honoraria: Roche International

Consulting or Advisory Role: Roche International

Selim Gebran

No relationship to disclose

Anthony Gebran

No relationship to disclose

Walid Ammar

No relationship to disclose

Affiliations

Fadia Elias, Ibrahim R. Bou-Orm, and Walid Ammar, Ministry of Public Health, Lebanon; Fadia Elias and Walid Ammar, Lebanese University; Salim M. Adib, Selim Gebran, Anthony Gebran, and Walid Ammar, American University of Beirut, Beirut, Lebanon; and Ibrahim R. Bou-Orm, Queen Margaret University, Edinburgh, United Kingdom.

\section{REFERENCES}

1. Abou-Daoud KT: Morbidity from cancer in Lebanon. Cancer 19:1293-1300, 1966

2. Ministry of Public Health - Lebanon: National Cancer Registry (2015). https://www.moph.gov.lb/ en/Pages/2/7164/national-cancer-registry

3. Ammar W: Health System and Reform in Lebanon. World Health Organization, Beirut, Lebanon. 2003

4. Elias F, Khuri FR, Adib SM, et al: Financial burden of cancer drug treatment in Lebanon. Asian Pac J Cancer Prev 17:3173-3177, 2016

5. IMS Institute for Healthcare Informatics: Global Oncology Trend Report: A Review of 2015 and Outlook to 2020. https://morningconsult.com/wp-content/uploads/2016/06/IMS-InstituteGlobal-Oncology-Report-05.31.16.pdf

6. Ledford H: Immunotherapy's cancer remit widens. Nature 497:544, 2013

7. Medicare Payment Advisory Commission: Medicare Part B Drugs and Oncology. http://www. medpac.gov/docs/default-source/congressional-testimony/071306_testimony_part-b_oncology. pdf?sfursn=0

8. Medicare Payment Advisory Commission: A Data Book: Health Care Spending and the Medicare Program, June 2017. http://medpac.gov/docs/default-source/data-book/jun17_ databookentirereport_sec.pdf

9. Republic of Lebanon, Ministry of Public Health: Statistical Bulletins. https://www.moph.gov.lb/ en/Pages/8/327/statistical-bulletins

10. Mailankody S, Prasad V: Five years of cancer drug approvals: Innovation, efficacy, and costs. JAMA Oncol 1:539-540, 2015

11. Kumar H, Fojo T, Mailankody S: An appraisal of clinically meaningful outcomes guidelines for oncology clinical trials. JAMA Oncol 2:1238-1240, 2016

12. PLoS Medicine Editors: Drug companies should be held more accountable for their human rights responsibilities. PLoS Med 7:e1000344, 2010

13. American Society of Clinical Oncology: American Society of Clinical Oncology Position Statement on Addressing the Affordability of Cancer Drugs. J Oncol Pract [epub ahead of print on November $1,2017]$

14. Goldstein DA, Clark J, Tu Y, et al: Global differences in cancer drug prices: A comparative analysis. J Clin Oncol 34, 2016 (suppl: abstr LBA6500) 
15. Prasad V, De Jesús K, Mailankody S: The high price of anticancer drugs: Origins, implications, barriers, solutions. Nat Rev Clin Oncol 14:381-390, 2017

16. Fricker J: New NICE criteria for drug access. Lancet Oncol 18:576, 2017

17. Chopra R, Lopes G: Improving access to cancer treatments: The role of biosimilars. J Glob Oncol 3:596-610, 2017

18. Republic of Lebanon, Ministry of Public Health: National Cancer Treatment Guidelines (2012). https://www.moph.gov.Ib/userfiles/files/Quality\%26Safety/NationalGuidelines/ 16UNDPcancerbook2012web.pdf 